\title{
Sequenced Chemotherapy and Surgery for Potentially Resectable Colorectal Liver Metastases: A Debate Over Goals of Research and an Approach While the Jury Remains Out
}

\author{
Jean-Nicolas Vauthey, $\mathrm{MD}^{1}$, Bernard Nordlinger, $\mathrm{MD}^{2}$, Scott Kopetz, $\mathrm{MD}^{3}$, and Graeme Poston, $\mathrm{MD}^{4}$ \\ ${ }^{1}$ Department of Surgical Oncology, The University of Texas MD Anderson Cancer Center, Houston, TX; ${ }^{2}$ Department of \\ Surgery and Oncology, Centre Hospitalier Universitaire Ambroise Paré, Boulogne-Billancourt, France; ${ }^{3}$ Department of \\ Medical Oncology, The University of Texas MD Anderson Cancer Center, Houston, TX; ${ }^{4}$ Aintree University Hospitals, \\ Liverpool, UK
}

The combination of hepatic resection and chemotherapy is increasingly accepted as an effective treatment for patients with hepatic colorectal metastases with surgical series reporting 5-year survival rates in excess of 50\% ${ }^{1-3}$ However, controversy remains regarding the surgery-chemotherapy sequence and the type of regimens to be used in combination with surgery. Further studies can go in 1 of 2 directions: either simplification of the treatment approach (comparison between preoperative versus postoperative chemotherapy) with the goal of reducing complications or intensification of treatment (comparison of perioperative chemotherapy regimens combining cytotoxic chemotherapy and biologic therapy) with the hope of improving survival. ${ }^{4}$ The main debate now is which question is most worthy of an answer and what do we do while the jury is out?

\section{WHAT QUESTIONS REMAIN TO BE ANSWERED?}

While the EORTC trial recently indicated that the sequence of chemotherapy followed by surgery can be used with favorable results and reduces the risk of cancer relapse after surgery, there has been concern regarding the effects of such preoperative chemotherapy on morbidity associated with subsequent surgery. ${ }^{4,5}$ The results of this trial demonstrated an increase in reversible postoperative

\footnotetext{
(C) Society of Surgical Oncology 2010
}

First Received: 13 July 2009;

Published Online: 16 March 2010

J.-N. Vauthey, MD

e-mail: jvauthey@mdanderson.org complications in 40 of 158 perioperative chemotherapy patients $(25 \%)$ versus 27 of 170 in surgery-alone patients $(16 \%)(P=0.04) .{ }^{5}$ While we and others advocating perioperative chemotherapy are concerned about an increase from 4 of 170 (2\%) to 11 of 159 (7\%) of postoperative intra-abdominal infections in the perioperative chemotherapy arm, increases from 5\% (8 of 170) to 7\% (11 of 159 ) of posthepatectomy hepatic failure, and $2 \%$ (3 of 170) to $3 \%$ (5 of 139) for reoperation following perioperative chemotherapy do not mitigate against such a protocol. Regardless, postoperative morbidity rates of $26 \%$ (seen in the neoadjuvant arm of the EORTC study) compare favorably to those rates seen following surgery alone from other single-center series, which have been reported as high as $34 \%{ }^{6}$ Furthermore, postoperative mortality was low in both arms of the study (1\%).

Also of relevance are the detailed pathologic studies describing chemotherapy specific associated hepatic injuries in patients subsequently coming to liver surgery with curative intent. One early study suggested an association between 5-fluorouracil and steatosis. ${ }^{7}$ More recently irinotecan has been associated with steatohepatitis, while oxaliplatin has been associated with sinusoidal injury. ${ }^{8}$ Series that have specifically studied pathological injuries have indicated an increased postoperative mortality from steatohepatitis in association with irinotecan. ${ }^{8,9}$ Increased postoperative complications, and perhaps increased perioperative bleeding, have been reported following prolonged preoperative use of oxaliplatin (median duration more than 4 months), and this risk was dependant on the number of cycles of chemotherapy ${ }^{10-12}$ As such, further evaluation of the perioperative risks of chemotherapy 
should reflect the current consensus that such treatment should optimally be 2-3 months in duration for potentially resectable patients.

As we move from surgical criteria alone for resection and increasingly select patients based on response to chemotherapy, an increasingly controversial issue is whether patients with resectable colorectal metastases should be taken promptly to the operating room or treated prior to surgical resection with systemic chemotherapy. One retrospective study has suggested that response to preoperative chemotherapy for multiple $(\geq 5)$ but resectable colorectal liver metastases may be a surrogate biological marker for prognosis. In this study, only $8 \%$ of patients who underwent resection while progressing on chemotherapy survived 5 years after hepatic surgery, compared with approximately $35 \%$ of those with disease response or stabilization. ${ }^{13}$ Interestingly, in the EORTC trial, the "open and close" rate was $5 \%$ (8 patients) in the perioperative chemotherapy arm that compared favorably to $11 \%$ (18 patients) in the surgery-alone arm, suggesting that neoadjuvant chemotherapy may help to positively select for resectability. ${ }^{5}$ While this observation is no more than a reflection of our opinion, it is likely that this increased rate of open and close in the surgery alone arm represents the reality of what happens in the community, when patients are taken immediately to the operating room without the benefit of a period of observation afforded by chemotherapy. This approach to selection for liver resection recognizes that the biology of the tumor, as defined by chemotherapy responsiveness and extent of subclinical metastases, is the primary determinant of long-term patient outcomes after hepatic resection.

As the safety of resection after appropriate chemotherapy is increasingly recognized, so are the critical aspects of its tenets, which include chemotherapy type, chemotherapy duration, and interval between surgery and chemotherapy. The EORTC trial was developed in an era predating our current knowledge of chemotherapy-associated hepatic injury and the terms of this new paradigm. Entry required characterization of the liver lesion(s) by no more than single slice CT scanning, and exclusion of extra-hepatic lung disease by a chest x-ray. The EORTC protocol mandated surgery within 4 weeks of completion of chemotherapy. ${ }^{5}$ As such, this trial cannot be used as an argument against preoperative chemotherapy without considering the large body of the literature suggesting the safety and effectiveness of specific chemotherapy types administered for limited time periods before surgery. ${ }^{8,12,14,15}$ It is now clear that surgery should be the mainstay of treatment for hepatic colorectal metastases, and there is no evidence to support the place for "adjuvant surgery," which greatly affects the results of resections performed after extended preoperative chemotherapy.
Therefore, patients should not be treated until "maximal" or "complete" response and tumor "regrowth" prior to consideration of surgical resection (which in reality are retrospective and clinically meaningless assessments).

We believe that those patients whose disease is clearly resectable at detection should continue to go into prospective randomized trials that address issues that combine surgical resection with chemotherapy (such as the currently ongoing CRUK [Cancer Research United Kingdom] study and the BOS [Biologics Oxalipaltin and Surgery] trial-EORTC 40051 currently in planning phase). A trial that addresses neoadjuvant versus adjuvant chemotherapy would provide useful data on approaches to minimize complications. ${ }^{4}$ However, the trial addressing this question (NSABP C-11) will not report until halfway through the next decade at the earliest. Further, based on the EORTC data showing that $24 \%$ of patients assigned to receive additional postoperative chemotherapy did not receive any chemotherapy after surgery, it is likely that a randomized trial comparing preoperative versus postoperative chemotherapy will be associated with a substantial number of patients not receiving any chemotherapy in the postoperative chemotherapy arm on an intention to treat basis. ${ }^{5}$

\section{WHAT DO WE DO WHILE WE AWAIT THESE RESULTS?}

Because the definitions of resectability, borderline resectability, and unresectability are surgeon dependent and do not predict the ultimate resectability in the operating room or after chemotherapy, we propose a uniform approach to patients whose colorectal metastases are resectable and borderline resectable and are not being entered into prospective randomized trials. Clearly, patients who meet the entry criteria for trials currently open should be recruited directly into those trials. Patients presenting with multiple innumerable liver metastases will probably never come to resection. However, for all others, including patients with numerous multiple metastases or large metastases, resection should be considered after limited chemotherapy. These patients should be treated upfront effectively with the most active currently approved regimen at that cancer center for colorectal metastases. Recent data indicate that only 23 months of such chemotherapy can achieve major pathologic response in the majority of patients $(<50 \%$ viable tumor cells per tumor surface area) without additional gain in response to more prolonged preoperative treatment. ${ }^{16,17} \mathrm{~A}$ proper evaluation after this short course of treatment appears appropriate, especially in patients with unresectable or borderline resectable metastases to determine whether patients at this point are candidates for resection or another second line regimen. ${ }^{18,19}$ If surgery is considered, patients should be 
evaluated for possible portal vein embolization if preoperative regeneration of the remnant liver anticipated after surgery appears to be indicated. In the absence of evidence of hepatic injury a future remnant liver of more than $20 \%$ appears to be sufficient for major resection. ${ }^{20}$ The interval between chemotherapy and surgery now becomes a critical tenet of postchemotherapy resectional surgery. Two recent prospective studies showed no increase in surgical complications and a favorable surgical outcome when cytotoxic chemotherapy alone, or chemotherapy with bevacizumab was discontinued 5 weeks prior to resection. ${ }^{14,21}$ As an alternative, others have recommended another cycle of cytotoxic chemotherapy without bevacizumab prior to surgery to extend this interval to $6-8$ weeks. ${ }^{15,22}$

Prior to surgery patients should be reimaged using triple-phase CT. In addition to shrinking lesions, biologic response to chemotherapy is also demonstrated in the form of loss of arterial phase enhancement and lesions with better defined borders than those seen at presentation. These morphologic changes do not indicate necrosis but replacement of tumor by fibrosis. ${ }^{16,23}$ In patients with a major or complete pathologic response, the cysticlike appearance defines replacement of the tumor surface area by fibrosis, independent of a decrease in lesion size. Indeed, most complete pathologic responses are associated with residual radiographic findings. New radiologic response criteria for patients treated with biologic agents for hepatic colorectal metastases have recently been defined. ${ }^{24}$ At this time, patients are candidates for resection, and the surgical mapping defining the extent of the planned resection should be based on the prechemotherapy imaging to encompass all metastatic sites, given the low complete pathologic response rate in lesions with complete radiographic response. ${ }^{25}$ In most patients with extensive bilateral metastases, 2-stage resection (most commonly partial left hepatectomy at the first operation, and then major right or extended right hepatectomy at a second operation following appropriate liver regeneration) should be preferred to a 1-stage procedure with radiofrequency ablation. ${ }^{26,27}$

Following surgical resection, patients remain candidates for postoperative (adjuvant) chemotherapy. The interval between surgery and postoperative chemotherapy remains to be clearly defined. In patients with problematic wound healing or wound infection, this interval should be carefully evaluated and extended. The return of serum bilirubin levels and hepatic transaminases to baseline should be considered prior to resuming chemotherapy. Elevated serum alkaline phosphatase is an indicator of hepatic regeneration and will remain high in some patients for some months following liver resection. Resuming bevacizumab should be avoided when there is poor wound healing.
As the learning curve following perioperative chemotherapy evolves, more data will accumulate regarding specific treatment sequences and approaches for patients with synchronous presentation of primary colorectal cancer with liver metastases, which remain less well defined. We advocate that patients with small solitary liver metastases $(<2 \mathrm{~cm})$ undergo immediate surgery for their liver lesion because of the risk of disappearance of these lesions with preoperative chemotherapy. Patients with multiple bilobar metastases or synchronous presentation and an isolated contralateral small metastasis with extensive disease on the other side of the liver might still benefit from preoperative chemotherapy, with the small lesion in the contralateral liver being marked with clips prior to chemotherapy by an interventional radiologist. ${ }^{28}$ As preoperative therapy has evolved as an important paradigm in the treatment of locally advanced rectal cancer, esophageal cancer, and breast cancer, patients with resectable and borderline resectable colorectal liver metastases should be given the ability to choose between $25 \%$ reduction in relapse rate (8\% gain in disease-free survival) and a modest (9\%) increase in reversible surgical complications without an increase in postliver resection mortality. Given the meaningful benefits that can be derived from hepatic resection, answering equally important questions in future trials will optimize patient outcome.

ACKNOWLEDGMENT The authors thank Dr. Daria Zorzi and Ms. Ruth Haynes for their contribution to the manuscript.

\section{REFERENCES}

1. Pawlik TM, Scoggins CR, Zorzi D, Abdalla EK, Andres A, Eng $\mathrm{C}$, et al. Effect of surgical margin status on survival and site of recurrence after hepatic resection for colorectal metastases. Ann Surg. 2005;241:715-24.

2. Abdalla EK, Vauthey JN, Ellis LM, et al. Recurrence and outcomes following hepatic resection, radiofrequency ablation, and combined resection/ablation for colorectal liver metastases. Ann Surg. 2004;239:818-25.

3. Fernandez FG, Drebin JA, Linehan DC, et al. Five-year survival after resection of hepatic metastases from colorectal cancer in patients screened by positron emission tomography with F-18 fluorodeoxyglucose (FDG-PET). Ann Surg. 2004;240:438-47.

4. Petrelli NJ. Perioperative or adjuvant therapy for resectable colorectal hepatic metastases. J Clin Oncol. 2008;26:4862-3.

5. Nordlinger B, Sorbye H, Glimelius B, et al. Perioperative chemotherapy with FOLFOX4 and surgery versus surgery alone for resectable liver metastases from colorectal cancer (EORTC Intergroup trial 40983): a randomised controlled trial. Lancet. 2008;371:1007-16.

6. Metreveli RE, Sahm K, Denstman F, et al. Hepatic resection at a major community-based teaching hospital can result in good outcome. Ann Surg Oncol. 2005;12:133-7.

7. Moertel CG, Fleming TR, Macdonald JS, et al. Hepatic toxicity associated with fluorouracil plus levamisole adjuvant therapy. $J$ Clin Oncol. 1993;11:2386-90. 
8. Vauthey JN, Pawlik TM, Ribero D, et al. Chemotherapy regimen predicts steatohepatitis and an increase in 90-day mortality after surgery for hepatic colorectal metastases. J Clin Oncol. 2006;24: 2065-72.

9. Fernandez FG, Ritter J, Goodwin JW, et al. Effect of steatohepatitis associated with irinotecan or oxaliplatin pretreatment on resectability of hepatic colorectal metastases. J Am Coll Surg. 2005;200:845-53.

10. Nakano H, Oussoultzoglou E, Rosso E, et al. Sinusoidal injury increases morbidity after major hepatectomy in patients with colorectal liver metastases receiving preoperative chemotherapy. Ann Surg. 2008;247:118-24.

11. Aloia T, Sebagh M, Plasse M, et al. Liver histology and surgical outcomes after preoperative chemotherapy with fluorouracil plus oxaliplatin in colorectal cancer liver metastases. J Clin Oncol. 2006;24:4983-90.

12. Karoui M, Penna C, Amin-Hashem M, et al. Influence of preoperative chemotherapy on the risk of major hepatectomy for colorectal liver metastases. Ann Surg. 2006;243:1-7.

13. Adam R, Pascal G, Castaing D, et al. Tumor progression while on chemotherapy: a contraindication to liver resection for multiple colorectal metastases? Ann Surg. 2004;240:1052-61; discussion 1061-4.

14. Gruenberger T, Sorbye H, Debois M, et al (2006) Tumor response to preoperative chemotherapy with FOLFOX-4 for resectable colorectal cancer liver metastases. Interim results of EORTC Intergroup randomized phase III study 40983. J Clin Oncol 24: 3500A.

15. Kesmodel SB, Ellis LM, Lin E, et al. Preoperative bevacizumab does not significantly increase postoperative complication rates in patients undergoing hepatic surgery for colorectal cancer liver metastases. J Clin Oncol. 2008;26:5254-60.

16. Ribero D, Wang H, Donadon M, et al. Bevacizumab improves pathologic response and protects against hepatic injury in patients treated with oxaliplatin-based chemotherapy for colorectal liver metastases. Cancer. 2007;110:2761-7.

17. Blazer DG III, Kishi Y, Maru DM, Kopetz S, Chun YS, Overman MJ, et al. Pathologic response to preoperative chemotherapy: a new outcome end point after resection of hepatic colorectal metastases. J Clin Oncol. 2008;26:5344-51.
18. Adam R, Aloia T, Levi F, et al. Hepatic resection after rescue cetuximab treatment for colorectal liver metastases previously refractory to conventional systemic therapy. J Clin Oncol. 2007; 25:4593-602.

19. Kopetz S, Vauthey JN. Perioperative chemotherapy for resectable hepatic metastases. Lancet. 2008;371:963-5.

20. Ribero D, Abdalla EK, Madoff DC, et al. Portal vein embolization before major hepatectomy and its effects on regeneration, resectability and outcome. Br J Surg. 2007;94:1386-94.

21. Welsh FK, Tilney HS, Tekkis PP, et al. Safe liver resection following chemotherapy for colorectal metastases is a matter of timing. Br J Cancer. 2007;96:1037-42.

22. Ellis LM, Curley SA, Grothey A. Surgical resection after downsizing of colorectal liver metastasis in the era of bevacizumab. J Clin Oncol. 2005;23:4853-5.

23. Rubbia-Brandt L, Giostra E, Brezault C, et al. Importance of histological tumor response assessment in predicting the outcome in patients with colorectal liver metastases treated with neoadjuvant chemotherapy followed by liver surgery. Ann Oncol. 2007;18:299-304.

24. Chun YS, Vauthey JN, Boonsirikamchai P, et al. Association of computed tomography morphologic criteria with pathologic response and survival in patients treated with bevacizumab for colorectal liver metastases. JAMA 2009;302:2338-44.

25. Benoist S, Brouquet A, Penna C, et al. Complete response of colorectal liver metastases after chemotherapy: does it mean cure? J Clin Oncol. 2006;24:3939-45.

26. Chun YS, Vauthey JN, Ribero D, et al. Systemic chemotherapy and two-stage hepatectomy for extensive bilateral colorectal liver metastases: perioperative safety and survival. J Gastrointest Surg. 2007;11:1498-504.

27. Aloia TA, Vauthey JN, Loyer EM, et al. Solitary colorectal liver metastasis: resection determines outcome. Arch Surg. 2006;141: $460-7$.

28. Zalinski S, Abdalla EK, Mahvash A, et al. A marking technique for intraoperative localization of small liver metastases before systemic chemotherapy. Ann Surg Oncol. 2009;16:1208-11. 